\title{
Lead Site Axial
}

National Cancer Institute

\section{Source}

National Cancer Institute. Lead Site Axial. NCI Thesaurus. Code C135390.

A bipolar electrocardiog ram limb lead which records the voltage between the positive electrode near the apex of the heart (located in the ICS of left 5-6 rib close to the sternum) and the neg ative electrode behind the right ear near the right mastoid process.

(CDISC) 